\title{
METEOROIDS AND METEOR STREAMS
}




\title{
THE ORBITAL DISTRIBUTION AND ORIGIN OF METEOROIDS
}

\author{
DUNCAN STEEL \\ Department of Physics and Mathematical Physics \\ The University of Adelaide \\ G.P.O. Box 498, Adelaide, SA 5001 \\ Australia
}

\begin{abstract}
Approximately 68,000 orbits of meteoroids, ranging from sizes of 10 $\mathrm{cm}$ and more down to microgram masses, are now available through the IAU Meteor Data Center. These orbits were measured in surveys based in the U.S.S.R., the U.S.A., Canada, Somalia, and Australia, using photographic, radar and television techniques; the data represent our best knowledge of the orbital distributions of smaller solid bodies in the solar system. It is found that quite different distributions result in different mass regimes, with implications for the origin and evolution of these particles: for example the larger bodies, observed as fireballs, are associated with meteorites in coming from the region of the asteroid belt with low-inclination orbits, whereas the smaller meteoroids have more comet-like orbits. There is also evidence for several meteoroid streams associated with specific Apollo asteroids. The data may additionally be viewed as a suitable source function in investigations of the production of interplanetary dust from the fragmentation of larger meteoroids in mutual collisions. However, inspection of the data raises many questions: for instance there seem to be many meteoroids on small retrograde paths, but no possible parent objects are known to exist on such orbits.
\end{abstract}

\section{Introduction}

Over the past decades, many different observational programs have been set up in which meteor orbits have been determined using a variety of techniques: starting in the 1930's using cameras with rotating shutters initially, and later, starting in the late 1940's but not reaching real fruition until the 1960's, using radar techniques which allow 24 hour coverage independent of weather conditions. In more recent years TV apparatus and other electronic detectors have been used (Jones and Sarma, 1985; Hawkes and Jones, 1986, and papers cited therein), and modern computing techniques have allowed the development of more sophisticated radars (Steel and Baggaley, 1985). Whilst some lists of individual orbits have been published (e.g. Jones and Sarma, 1985), in some cases not all of the orbital elements have been given $(e . g$. Lebedinets $e t a l, 1981,1982)$ and in other cases only the results of stream searches have been published (e.g. Sekanina, 1976). In any case, for some surveys many thousands of orbits have been determined so that

A.C. Levasseur-Regourd and H. Hasegawa (eds.), Origin and Evolution of Interplanetary Dust, 291-298. (C) 1991 Kluwer Academic Publishers, Printed in Japan. 
the data are required by other researchers in a machine-readable form rather than on paper, if later independent analysis is to be feasible.

In order that this data resource might be fully exploited, a Meteor Data Center was set up at the Lund Observatory in Sweden with sponsorship from the IAU, under the direction of B.-A. Lindblad. All available meteor orbit data have been collected together, converted into a common format, checked for consistency of the elements and the given velocities and radiants, and the missing elements (such as the perihelion distance, the argument of perihelion, or the nodal longitude) have been filled in for each orbit; this task was largely carried out by the author whilst on an ESA Fellowship in Lund. The full data set, which is still being added to as other data (such as fireball orbits from the European Network) become available, may be obtained by any interested researcher. The presently-available data are as follows, a brief description also having been given by Lindblad (1987):

\section{Survey name}

Harvard graphical photographic meteors

Harvard precise photographic meteors

Prairic Network fireballs

Meteorite Orbit and Recovery Program fireballs

Soviet photographic meteors

Television meteors

Harvard radar meteors 1961-65

Harvard radar meteors $1968-69$

Obninsk radar meteors

Mogadisho (Somalia) radar meteors

Kharkov radar meteors

Adelaide radar meteors 1960-61

Adelaide radar meteors 1968-69

\section{Country Number}

$\begin{array}{lr}\text { USA } & 2529 \\ \text { USA } & 1245 \\ \text { USA } & 336 \\ \text { Canada } & 218 \\ \text { USSR } & 1111 \\ \text { Canada } & 531 \\ \text { USA } & 19327 \\ \text { USA } & 19818 \\ \text { USSR } & 9358 \\ \text { USSR } & 5328 \\ \text { USSR } & 5317 \\ \text { Australia } & 2092 \\ \text { Australia } & 1667\end{array}$

Since some Harvard photographic meteors were reduced both precisely and graphically, the total number of independent meteor orbits available from the above is about 68,000 . The data are of variable quality, and in checking the orbits it was found that in some surveys $10 \%$ or more were clearly in error; for example one particular element not fitting with the others, but doing so if a typographical error in one digit were corrected. Additionally, the different surveys pertain to rather differently sized meteoroids: the photographic surveys detect mostly the meteors from particles which were originally of mass $1 \mathrm{mg}$ and larger (sizes rather greater than $1 \mathrm{~mm}$ ) whilst the radar surveys (plus the TV meteors) are dominated by meteoroids smaller than this; for the Harvard and the Kharkov radar surveys, which had the lowest limiting magnitudes, masses down to micrograms.

\section{Usefulness of these data}

Several points can be made concerning the distribution of meteoroids in the inner solar system, and what such information can tell us about their origin and evolution. The data can be used for specific searches for streams, and also their association with certain parent objects. For example, Olsson-Steel (1988) 
used the Adelaide orbits to show that there are several Apollo-type asteroids which apparently have spawned meteoroid streams, so that it is not purely comets which generate meteoroids (unless the asteroids are defunct cometary cores). In addition, one of the major sources of the zodiacal dust is the catastrophic destruction of meteoroids (sizes $100 \mu \mathrm{m}-10 \mathrm{~cm}$ ) in impacts with smaller particles (mainly $10 \mu \mathrm{m}-100 \mu \mathrm{m}$ ) so as to re-generate this cloud as it decays under other effects (e.g. Grun et al, 1985). In order to attack this problem, the regions where dust is generated needs to be known, along with the collisions velocities and other parameters. The meteoroid data described herein provide a suitable source function for such investigations since the spatial densities and thus the collision frequencies can be calculated $(e . g$. Kneissel and Giese, 1987).

\section{New meteor orbits}

Whilst there is a wealth of meteor orbit data available, it is also necessary to collect new orbits for a number of reasons, for instance searching for newlyappeared showers, or checking on the variation of stream parameters as they evolve. There are several amateur groups world-wide which are engaged in photographic observations which lead to excellent meteor orbits, notably in northern Europe and in Japan (Lindblad, this volume).

In addition it is notable that comparatively few southern hemisphere orbits have been collected; the Adelaide radar surveys, rendering less than 4,000 orbits in total, have been the only programs based in the southern hemisphere, although the Soviet expedition to Mogadisho also resulted in many southern radiants being measured. It is thus noteworthy that a new radar survey has begun in New Zealand under the direction of W.J.Baggaley. The equipment and techniques were described by Steel and Baggaley (1985). Routine data collection began in November 1989, with two days data collection occurring every month. Since approximately 2,000 orbits are determined in each 24-hour run, this survey has already resulted in more individual meteor orbits than the Harvard radar program listed above.

\section{Example of meteor orbit data}

As an example of the sorts of data which are available, the Obninsk radar survey (Lebedinets et al, 1981, 1982) is chosen here. This survey had a limiting radar magnitude of +7.5 . The reasons for this choice are: (i) There is a large number of individual orbits available; (ii) The meteoroid sizes here are approximately $1 \mathrm{~mm}$, this being about the mid-point between the larger photographic meteors and the smaller particles detected in the Harvard and Kharkov surveys; (iii) There is clear evidence of several streams in the data; and (iv) The data have been found to be of very high quality, with comparatively few errors. The Adelaide orbits are of similar quality, and some element distribution plots have been presented by Olsson-Steel (1988). Here a more extensive set will be given for the Obninsk data, similar sets for all other surveys listed above being given by Steel and Lindblad (1991).

In Figure 1 we show the distributions of the orbital elements $a, e, q, Q, i$ and $\omega$, where the symbols have their usual meanings. The number on each plot 

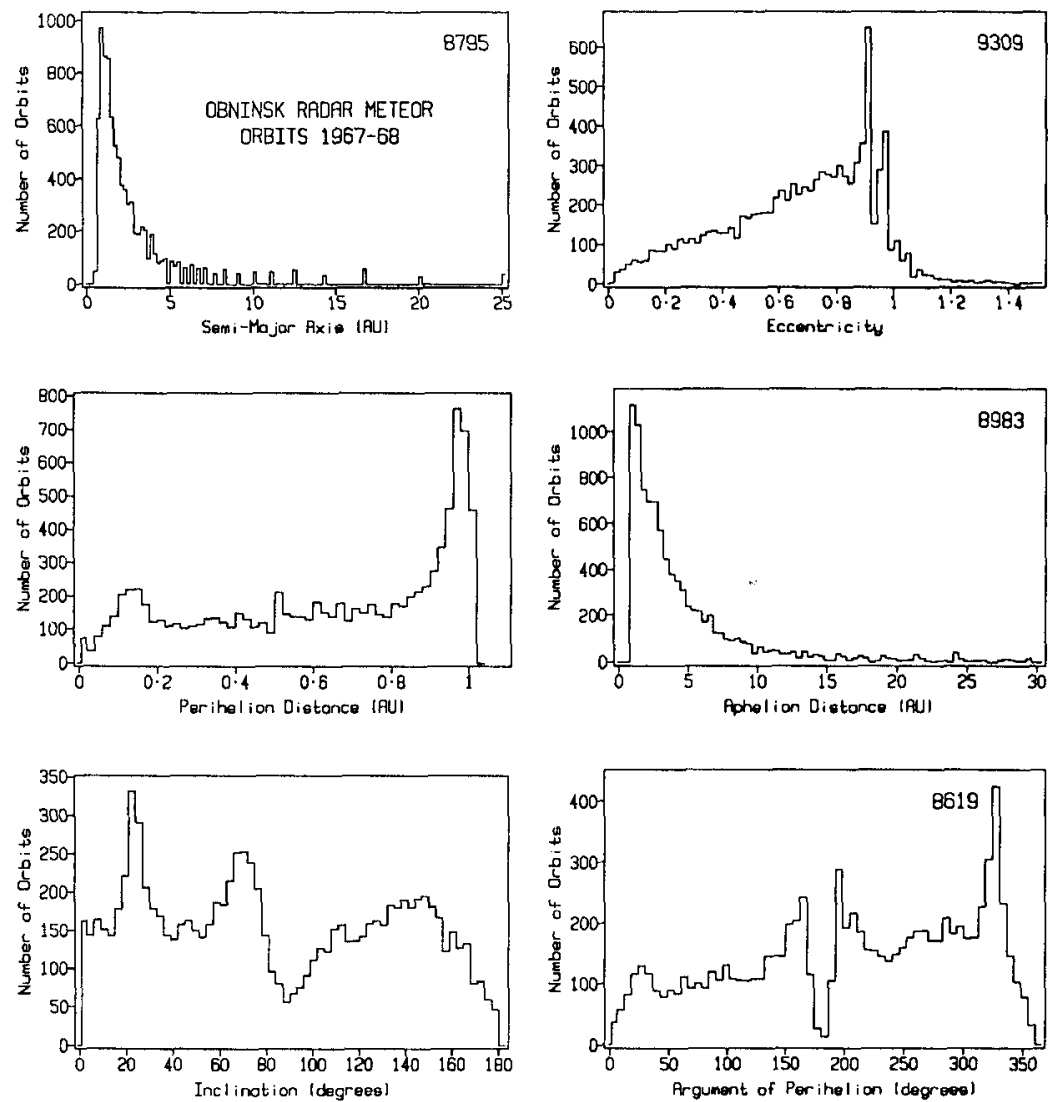

Figure 1. Distributions of orbital elements $a, e, q, Q, i$ and $\omega$ from the Obninsk radar meteor survey (Lebedinets et al, 1981, 1982).

indicates the total number of meteors contributing; for example for $a$, of the 9358 orbits in the entire survey only 8795 had $a<=25 \mathrm{AU}$. For $\omega$ it was necessary to calculate the value from the other elements, and this was not possible in some cases. The plot for $a$ clearly shows discrete larger values; this is because the semi-major axes were stored as the orbital energy $(1 / a)$ and this was only given to two decimal places. We note that the $a$ and $Q$ plots indicate that these meteoroids are in quite small orbits, with aphelia well within Jupiter: these are not, in this sense, by any means 'cometary'. However, they are largely on high-eccentricity orbits: the plot for $e$ shows a general increase, with structure at $e>0.8$ being due to the presence of various streams. In particular the Geminids have $e \cong 0.9$, and these are further evidenced by the bump at $q \cong 0.15 \mathrm{AU}$ and at $i \cong 23^{\circ}$. We note also the apparent existence of some hyperbolic orbits, the reality of which has been a matter of some debate in the past (see e.g. Jones and Sarma, 1985). Unlike many other surveys, but similarly to the Harvard radar data, the inclination distribution does not show 
a preponderance of low- $i$ orbits, rather many high- $i$ and retrograde orbits being seen. This may be due to various selection effects which depend upon the velocities of the meteors, and the radiants compared to the antenna patterns. Thus there are few orbits with $i \cong 90^{\circ}$, since these would all have very northerly radiants and would additionally need to have $\omega \cong 0^{\circ}$, and these are not seen in the $\omega$ plot; similarly there are few orbits with $\omega \cong 180^{\circ}$ since these would tend to emanate from deep southerly radiants. Another selection effect is due to the collision probability with the Earth: although the $q$-plot shows a strong peak at $\mathrm{q} \cong 1 \mathrm{AU}$ this is due to such orbits having much higher collision probabilities, so that this distribution does not represent the true distribution in space.
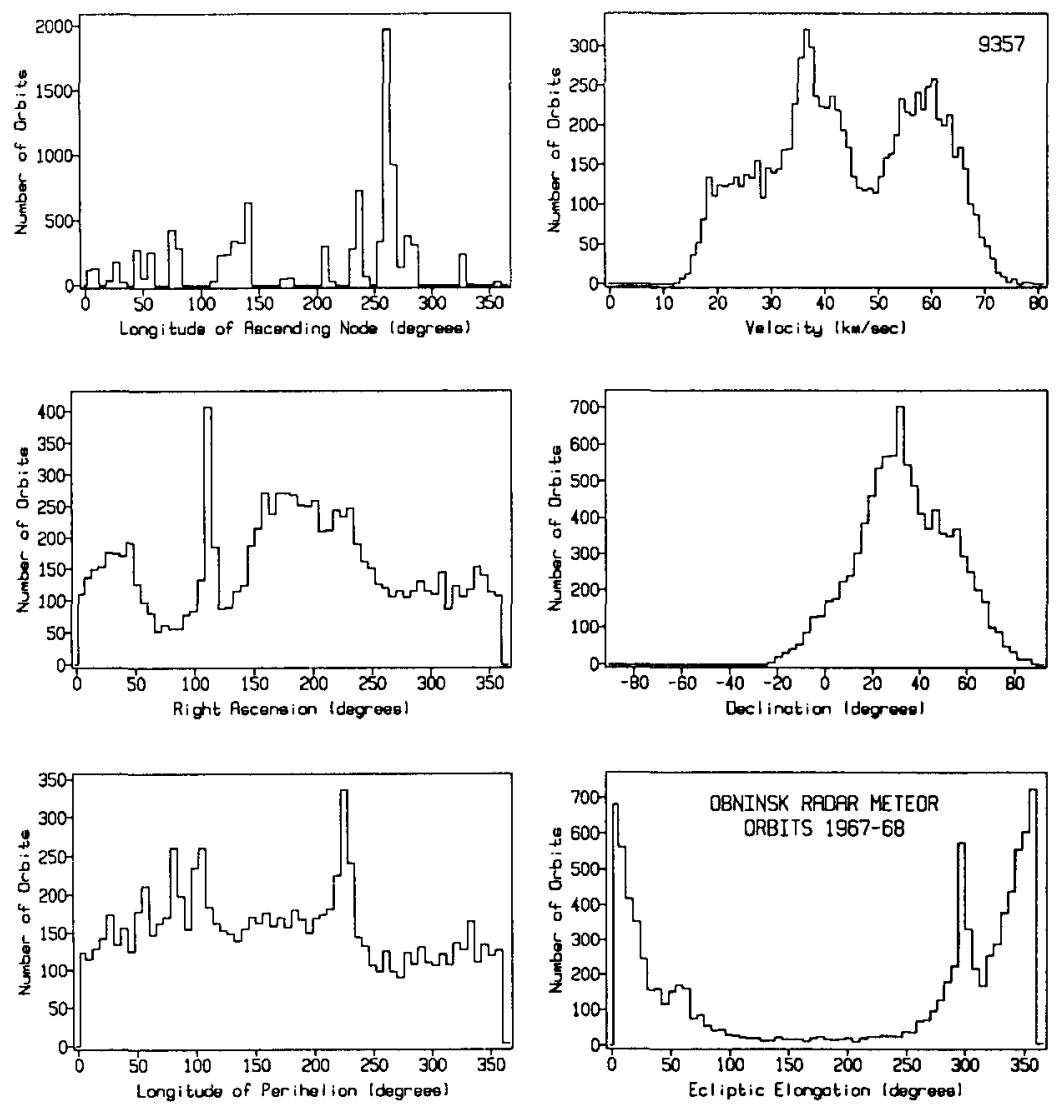

Figure 2. Distributions of various parameters of the Obninsk radar meteors.

In Figure 2 we show distributions for various other parameters of interest. The longitude of the ascending node $(\Omega)$ plot is very patchy, this being due to the distinct times of year in which observations were carried out and the number of meteors detected at each time; as pointed out below, these struck the Earth at their descending nodes. The peak near $\Omega=265^{\circ}$ is due to the Geminids, 
this shower also being apparent in the other plots. The velocity distribution shows twin peaks, as seen in other surveys, but here there are comparatively few low-velocity meteors: the peak at $V \cong 60 \mathrm{~km} / \mathrm{sec}$ is usually much less prominent. If there were no showers, and if the data collection were continuous and not patchy, then the RA distribution would be expected to be flat, and this is clearly not the case. As expected the Declination plot peaks near the zenithal value for this observation site. The Longitude of Perihelion is a useful parameter since it conveniently indicates the presence of showers amongst sporadic data, as here. Finally the plot of the Ecliptic Elongation ( $\varepsilon$ ) of the radiant is similar to that seen in other surveys, with peaks being evidenced at $\varepsilon \cong 0^{\circ}$ (Apex source), $60^{\circ}$ (Helion source) and $300^{\circ}$ (Anti-helion source), although the second of these is quite muted.
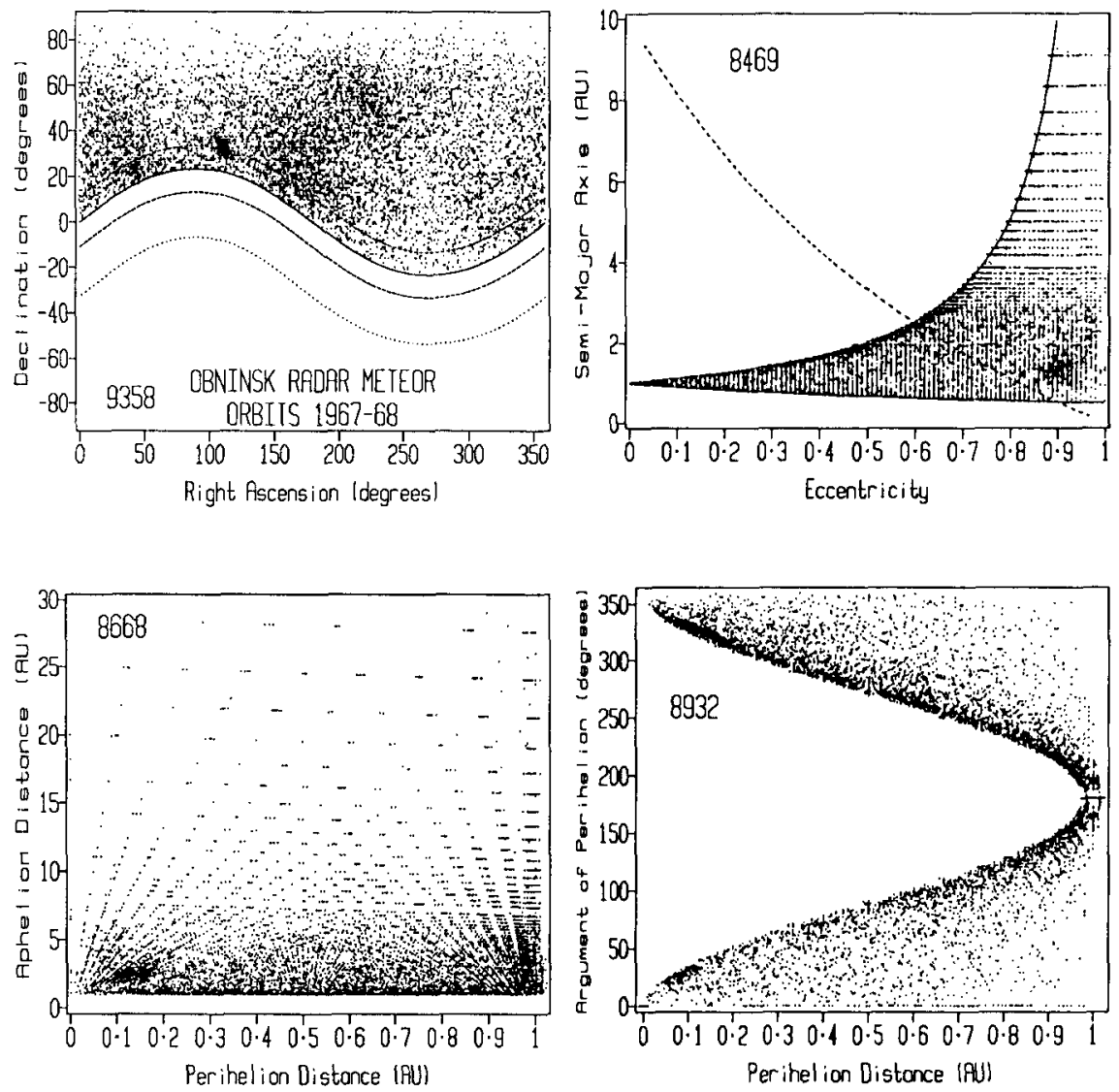

Figure 3. Radiant and orbital element distributions for the Obninsk data.

Figures 1 and 2 were just number distribution plots. We now present plots in which two parameters are plotted against each other in order to see what sort of information may be gained in this way. In Figure 3 we plot Declination 
versus RA, $a$ versus $e, Q$ versus $q$, and $\omega$ versus $q$. The Obninsk data which were published contain only radiants north of the ecliptic (with one exception, given in error) so that the first of these plots has no radiants from the south; this also means that these meteors were all observed at their descending node. There are several concentrations in this plot, due to showers, and in particular the Geminids stand out. For the $a$ versus $e$ plot three curved lines are shown. The two solid lines delineate the region for which Earth-intercept is possible (i.e. $q<1.0167 \mathrm{AU}$ and $Q>0.9833 \mathrm{AU}$ ); some orbits lie just beyond this region due to round-off errors. The dashed line shows Whipple's K-Criterion, orbits lying above it being classed as being 'cometary', those below it being 'asteroidal'. There is a more-or-less equal split, this being a useful indicator of the origin of these bodies. The Geminids are seen at $(a \cong 1.3 \mathrm{AU}, e \cong 0.9)$ in this plot, and in the $Q$ versus $q$ plot at $(q \cong$ $0.15 \mathrm{AU}, Q \cong 2.5 \mathrm{AU})$. In that plot it is notable that many of the orbits with $Q<5$ AU have $q>0.9 \mathrm{AU}$. In the final plot of Figure 3 the form of the scatter is due to these orbits being observed at the descending node only; for the ascending node a chevron opposing that plotted would be formed.
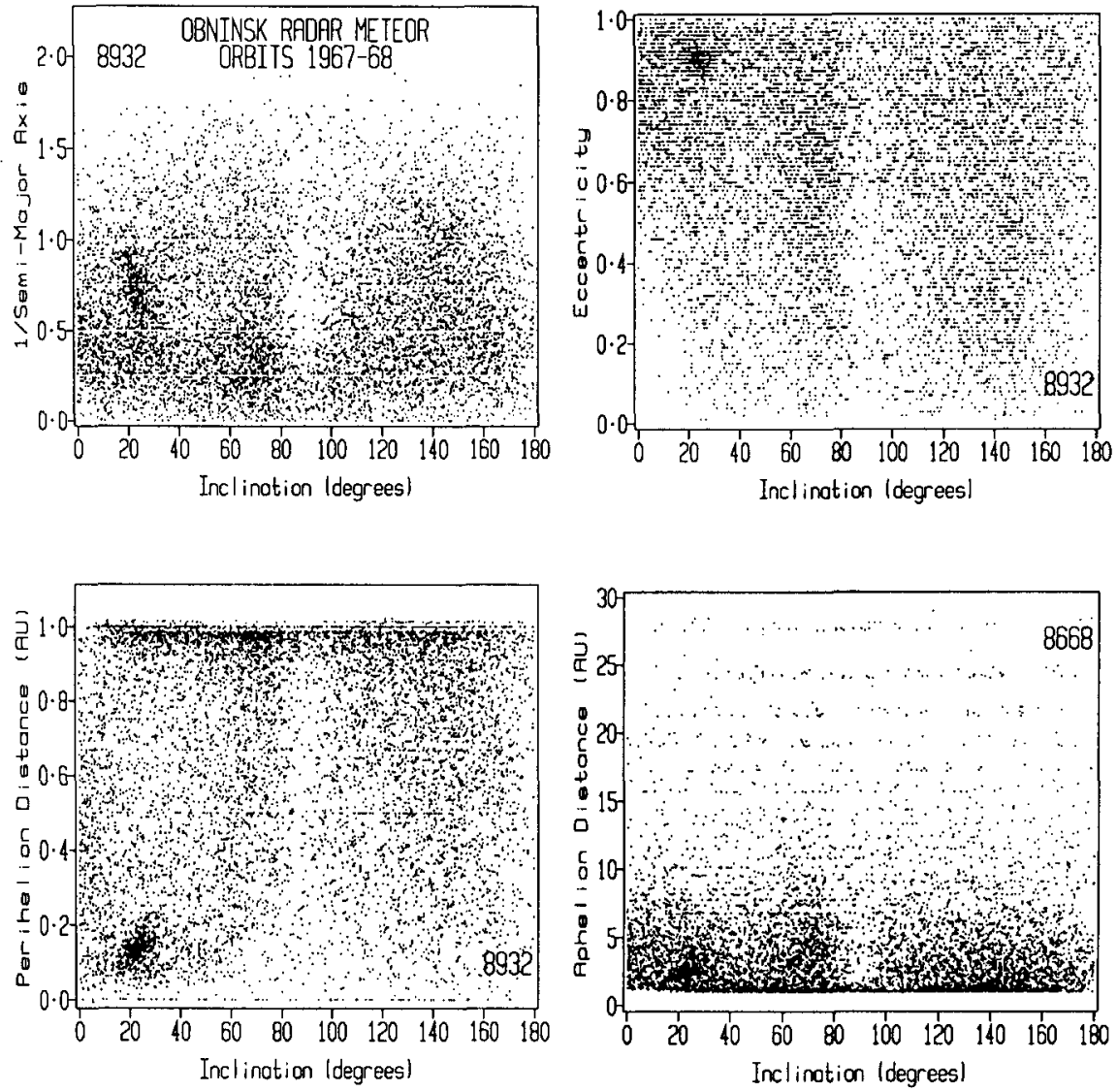

Figure 4. (1/a), $e, q$ and $Q$ plotted against $i$ for the Obninsk radar orbits. 
In Figure 4 we plot $(1 / a), e, q$ and $Q$ in turn against the inclination, $i$. The Geminids in particular are prominent in each plot. In the $(1 / a)$ plot we note that there are many meteoroids in retrograde Aten-type $(a<1 \mathrm{AU})$ orbits, for which no parent objects are known: these seem to be spread over all value of $q$ and $e$. Similar distributions are seen in other surveys so that the existence of such meteoroid orbits is certain. The question which then needs to be asked is: Were these meteoroids released onto such orbits by some as-yet undiscovered class of parent, or did they evolve onto these orbits under the influence of planetary perturbations/radiative effects/other influences? This dissimilarity to the known parent objects in the inner solar system is emphasized by the $Q$ versus $i$ plot, which shows no preponderance of retrograde meteors with large aphelia (i.e. these orbits are not like long-period comets, or $\mathrm{P} /$ Halley, P/Swift-Tuttle and the other known retrograde intermediate- or short-period comets).

Acknowledgements: This work was supported by the Australian Research Council and The University of Adelaide.

\section{References}

Grun, E., Zook, H.A., Fechtig, H. and Giese, R.H. (1985). 'Collisional balance of the meteoroitic complex', Icarus, 62, 244-272.

Jones, J. and Sarma, T. (1985). 'Double-station observations of 454 TV meteors. II. Orbits', Bull. Astron. Inst. Czechoslov., 36, 103-115.

Hawkes, R.L. and Jones, J. (1988). 'Electro-optical meteor observation techniques and results', Q. Jl. Roy. Astron. Soc., 27, 569-589.

Kneissel, B. and Giese, R.H. (1987). 'The dynamics of the zodiacal dust cloud on account of optical and infrared observations', Publ. Astron. Inst. Czechoslov. Acad. Sci., No. 67, vol. 2, 241-243.

Lebedinets, V.N., Korpusov, V.N. and Manokhina, A.V. (1981, 1982), 'Radio Meteor Investigations in Obninsk: Catalogue of Orbits', Materials of the World Data Center B, Moscow, U.S.S.R.

Lindblad, B.-A. (1987). 'The IAU Meteor Data Centre in Lund', Publ. Astron. Inst. Czechoslov. Acad. Sci., No. 67, vol. 2, 201-204.

Olsson-Steel, D. (1988). 'Identification of meteoroid streams associated with Apollo asteroids in the Adelaide radar orbit surveys', Icarus, 75, 64-96.

Sekanina, Z. (1976). 'Statistical model of meteor streams. IV. A study of radio streams from the synoptic year', Icarus, 27, 265-321.

Steel, D.I. and Baggaley, W.J. (1985). 'Meteoroid orbits determined by southern hemisphere radar', in Properties and Interactions of Interplanetary Dust, IAU Colloquium No. 85 (eds. R.H.Giese and Ph.Lamy), D.Reidel, Dordrecht, Holland, 299-303.

Steel, D.I. and Lindblad, B.-A. (1991). 'The meteor orbit database', Space Science Rev. (submitted). 